\title{
Electron Spin Resonance of defects in the Haldane System $\mathbf{Y}_{2} \mathrm{BaNiO}_{5}$
}

\author{
C.D. Batista, K. Hallberg and A.A.Aligia \\ Centro Atómico Bariloche and Instituto Balseiro \\ Comisión Nacional de Energía Atómica \\ 8400 S.C. de Bariloche, Argentina.
}

(Received February 26, 2018)

\begin{abstract}
We calculate the electron paramagnetic resonance (EPR) spectra of the antiferromagnetic spin-1 chain compound $\mathrm{Y}_{2} \mathrm{BaNi}_{1-x} \mathrm{Mg}_{x} \mathrm{O}_{5}$ for different values of $x$ and temperature $T$ much lower than the Haldane gap $(\sim 100 \mathrm{~K})$. The low-energy spectrum of an anisotropic Heisenberg Hamiltonian, with all parameters determined from experiment, has been solved using DMRG. The observed EPR spectra are quantitatively reproduced by this model. The presence of end-chain $S=1 / 2$ states is clearly observed as the main peak in the spectrum and the remaining structure is completely understood.

PACS numbers: $75.40 \mathrm{Cx}, 75.10 \mathrm{Jm}, 75.40 \mathrm{Mg}$.
\end{abstract}

A great deal of interest in one dimensional (1D) Heisenberg chains with nearest-neighbor antiferromagnetic exchange coupling, $J$, has been originated by Haldane's conjecture that integer-valued spin chains would exhibit a gap in the spin-wave excitation spectrum [1]. An interesting model of the underlying wave function for the Haldane state has been proposed by Affleck et al. [2]. They have shown that the exact ground state of the Hamiltonian $\sum_{i \mathbf{S}_{i} \cdot \mathbf{S} i+1}+\left(\mathbf{S}_{i} \cdot \mathbf{S}_{i+1}\right)^{2} / 3$ is a valence-bondsolid (VBS) state. This state in an open chain has two unpaired $S=1 / 2$ spins, one at each end. In agreement with this, exact diagonalization [3] of finite open Heisenberg chains (without the biquadratic term) shows that the four lowest-lying states are a triplet and singlet whose energy separation approaches zero exponentially with increasing length. Monte Carlo [4] and densitymatrix renormalization-group (DMRG) 河 studies clearly show the presence of $S=1 / 2$ end states.

This picture was supported by EPR measurements of $\left[\mathrm{Ni}\left(\mathrm{C}_{2} \mathrm{H}_{8} \mathrm{~N}_{2}\right)_{2}\left(\mathrm{NO}_{2}\right)\right] \mathrm{ClO}_{4}$ (NENP) doped with nonmagnetic ions [7], where resonances corresponding to the fractional spin $S=1 / 2$ states at the "open" ends of the $S=1 \mathrm{Ni}$ chains were observed. Similar measurements for doping with magnetic ions are also consistent with $S=1 / 2$ end states [8]. This effect, if robust, would correspond to the only instance in magnetism where a low energy collective excitation has no classical analog.

However, Ramirez et al. [9] also tested the presence of free $S=1 / 2$ states by studying the specific heat of non-magnetic defects in $\mathrm{Y}_{2} \mathrm{BaNiO}_{5}$, with magnetic fields up to $9 \mathrm{~T}$ and temperatures down to $0.2 \mathrm{~K}$. They found that the shape and magnitude of the Schottky anomaly associated with the defects in $\mathrm{Y}_{2} \mathrm{BaNi}_{1-x} \mathrm{Zn}_{x} \mathrm{O}_{5}$ are better described by a simple model involving spin-1 excitations, instead of the $S=1 / 2$ excitations of the VBS. In order to eliminate the apparent discrepancy with the EPR measurements in NENP, Ramirez et al. [9] pointed out the possibility that a small fraction of ethylene diamine complexes in NENP acquire charge at structural defects induced by $\mathrm{Zn}$ doping. As it is not uncommon that structural defects induce a paramagnetic behavior in organic compounds, they propose that similar EPR measurements should be performed on $\mathrm{Y}_{2} \mathrm{BaNi}_{1-x} \mathrm{~A}_{x} \mathrm{O}_{5}$ (A a nonmagmetic ion) where $x$ is more easily calibrated [9]. EPR is an appropriate technique to determine the existence of $\mathrm{S}=1 / 2$ end states because it allows to distinguish between $S=1 / 2$ and $S=1$ spins in the presence of spatial anisotropy.

In a recent paper [6], a precise fit of the above mentioned specific heat measurements was done, solving the low-energy spectrum of an anisotropic Heisenberg Hamiltonian for $\mathrm{Y}_{2} \mathrm{BaNi}_{1-x} \mathrm{Zn}_{x} \mathrm{O}_{5}$. It was shown that there is no contradiction between EPR in NENP [7] and specific heat measurements in $\mathrm{Y}_{2} \mathrm{BaNi}_{1-x} \mathrm{Zn}_{x} \mathrm{O}_{5}$ [9]. The results supported the existence of $S=1 / 2$ excitations for sufficiently long chains and clearly indicated that the anisotropy plays a very important role in the low temperature properties. However, EPR experiments would unambiguously detect the presence of such excitations and definitely confirm the validity of the theory.

Recently, Saylor et al [13] have measured the EPR spectra of $\mathrm{Y}_{2} \mathrm{BaNi}_{1-x} \mathrm{Mg}_{x} \mathrm{O}_{5}$ at temperature $T=2 K$ for different values of $\mathrm{Mg}$ concentration. The spectra show a prominent main peak, which can be associated with free $\mathrm{S}=1 / 2$ spins, surrounded by some secondary peaks. The appearance of the latter is not related with the interaction between the $S=1 / 2$ end states belonging to different chains. Thus, it is necessary to give an explanation for the origin of these other resonances.

In this paper, we calculate the EPR spectra of $\mathrm{Y}_{2} \mathrm{BaNi}_{1-x} \mathrm{Mg}_{x} \mathrm{O}_{5}$ at $T=2 \mathrm{~K}$ for different values of $x$ $(x=0.002,0.006$, and 0.02) and compare them with experiment. $\mathrm{Mg}$ and $\mathrm{Zn}$ are non-magnetic impurities, so the substitution of these ions for $\mathrm{Ni}$ provides a simple break in the chain. Due to the high sensitivity of EPR experiments, it is optimal for discerning between long chains (where the $S=1 / 2$ end spins are nearly independent) and the shorter chains (where the $S=1 / 2$ objects are overlapping). We use the same Hamiltonian as in Ref. [6], with the same parameters as those 
taken there from inelastic neutron scattering [10,11. The calculated EPR spectra show several contributions from chains with different lengths which are quantitatively reproduced. These contributions give rise to the secondary peaks mentioned above. The location of these peaks are related to the effect of anisotropy on chains with different lengths. In this way, these secondary peaks constitute clear evidence that the observed spectrum is originated by the end states of the $1 \mathrm{D} \mathrm{NiO}_{5}$ chains and not by paramagnetic impurities.

$\mathrm{Y}_{2} \mathrm{BaNiO}_{5}$ has an orthorhombic crystal structure with the $\mathrm{Ni}^{2+}(S=1)$ ions arranged in linear chains with a nearest-neighbor antiferromagnetic superexchange coupling $J$. The interchain coupling $J_{\perp}$ is at least three orders of magnitude weaker, making this compound an ideal one-dimensional antiferromagnetic chain. While each $\mathrm{Ni}$ atom is surrounded by six $\mathrm{O}$ atoms in near octahedron coordination, the true site symmetry is $D_{2 h}$. Neglecting $J_{\perp}$ (the effect of which will be discussed later), the appropriate Hamiltonian for a chain segment of length $N$ between two Mg impurities is 110 12]:

$$
\begin{aligned}
H= & \sum_{i}\left\{J \mathbf{S}_{i} \cdot \mathbf{S}_{i+1}+D\left(S_{i}^{z}\right)^{2}+E\left[\left(S_{i}^{x}\right)^{2}-\left(S_{i}^{y}\right)^{2}\right]\right\} \\
& -\mu_{B} \sum_{v \alpha} B^{\alpha} g^{\alpha \nu} S_{t}^{\nu}
\end{aligned}
$$

where $z$ is along the chain axis, $\mathbf{S}_{t}$ is the total spin and $g^{\alpha \nu}$ is the gyromagnetic tensor . Recent estimates based on fits of the Haldane gaps (in $x, y$, and $z$ directions) measured by inelastic neutron scattering, indicate $J \sim$ $280 K, D \sim-.038 J$, and $E \sim-.0127 J$ 10, 11. As $\mathrm{Y}_{2} \mathrm{BaNiO}_{5}$ has a Haldane gap $\sim 100 K$ [10,11, the spin wave contribution to the $\mathrm{EPR}$ is negligible at $2 K$. In this temperature range, EPR is dominated by the effect of the defects. For this reason, it is necessary to solve accurately the low energy spectra $(\omega \leq 10 K)$ of $H$ for all values of $N$ to be able to explain the observed EPR experiment.

The lowest energy states of Eq.(11) are a spin singlet, which we denote as $|0\rangle$, and three components of a split spin triplet $\left(\left|1 S_{t}^{z}\right\rangle\right)$. The energy of $|11\rangle$ is the same as that of $|1-1\rangle$ due to the time-reversal symmetry of $H$. By means of the DMRG method, we have calculated the energy of these four states for all $N \leq 40$ and $E=0$. The difference of energy $e$ between any pair of eigenstates decays exponentially to zero with increasing $N$. This behavior allows us to extrapolate the energies to all values of $N>40$, and demonstrate that the $S=1 / 2$ spins at the end of the chains are also asymptotically free in the presence of anisotropy. This issue is easy to understand considering that $S=1 / 2$ spins cannot be affected by anisotropy due to time-reversal symmetry. At this level, it is important to remark that the two end $S=1 / 2$ spin excitations, which are localized at the end sites in the VBS, have a finite localization length $l \sim 6$ sites for the pure Heisenberg model [3 5]. Therefore, while they are nearly free for large $(N>>l)$ open chains, the interaction between them is considerable when the length of the chain $N$ is comparable to $2 l$. This interaction splits the two $S=1 / 2$ states into a singlet (ground state for even $N$ ) and a triplet (ground state for odd $N$ ) [14].

The difference between any two energies of the above mentioned low-energy states is linear in $D$, and the quadratic corrections are negligible [12]. This widely justifies the validity of perturbation theory to first order in $D$. Then, by symmetry we can also include the term $\sum_{i} E\left[\left(S_{i}^{x}\right)^{2}-\left(S_{i}^{y}\right)^{2}\right]$ to first order. Thus we find the following low-energy effective Hamiltonian including the triplet $\left|1 S_{z}\right\rangle$ and the singlet state $|0\rangle$ :

$$
\begin{aligned}
H_{e f f}= & E_{0}(N)+(J \alpha(N)+D \beta(N))|0\rangle\langle 0|+D \gamma(N) S_{z}^{2} \\
& +E \gamma(N)\left(S_{x}^{2}-S_{y}^{2}\right)-\mu_{B} \sum_{\nu \alpha} B^{\alpha} g^{\alpha \nu} S_{t}^{\nu}
\end{aligned}
$$

where $E_{0}(N), \alpha(N), \beta(N)$ and $\gamma(N)$ are functions of the chain length $N$, determined from the DMRG data fitting exactly the four lowest energy levels for $E=0$. The numbers $\alpha(N), \beta(N)$ and $\gamma(N)$ are represented in Fig. 1. The validity of the last term of Eq.(2) has been verified explicitly by calculating the matrix elements of $S_{t}^{+}$, and $S_{t}^{-}$for all chains. $H_{\text {eff }}$ determines the thermodynamics of the system at temperatures well below the Haldane gap.
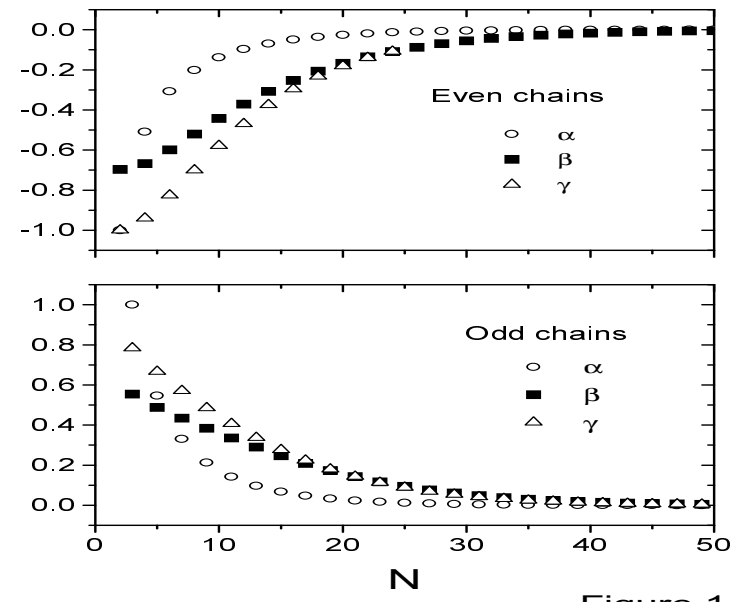

FIG. 1. Numerical coefficients of the effective Hamiltonian Eq. (2) as functions of the chain length.

Assuming a random distribution of defects, the probability to find a $\mathrm{Mg}$ atom at any site $i$ followed by $N \mathrm{Ni}$ sites and another $\mathrm{Mg}$ atom at site $i+N+1$ is clearly $x^{2}(1-x)^{N}$, where $x$ is the concentration of $\mathrm{Mg}$ (missing $S=1 \mathrm{Ni}$ spins). Thus, the probability per Mg impurity of finding a segment of $N \mathrm{Ni}$ atoms is $x(1-x)^{N}$. Then, the EPR signal intensity $I(x, \omega, T)$ per impurity is: 


$$
I(x, \omega, T)=\sum_{N=1}^{\infty} x(1-x)^{N} I_{N}(\omega, T)
$$

where $I_{N}(\omega, T)$ is the EPR signal intensity of a segment of length $N$ described by $H_{e f f}$. This intensity is given by the following expression:

$$
I_{N}(\omega, T) \propto \sum_{\alpha, \nu=1}^{4}|\langle\nu|\mathbf{S} \cdot \mathbf{n}| \alpha\rangle|^{2} e^{-\beta E_{\alpha}} \delta\left(\omega-E_{\nu}+E_{\alpha}\right)
$$

where $|\alpha\rangle$ are the eigenstates of $H$ (or $H_{e f f}$ ) for the $N$ site chain, $\mathbf{n}$ is the direction (orthogonal to $\mathbf{B}$ ) of the applied microwave magnetic field and $\beta$ the inverse temperature. The delta distributions have been replaced by Lorentzian functions with a finite width to simulate the effect of interactions not included in Eq.(2) and the experimental resolution.

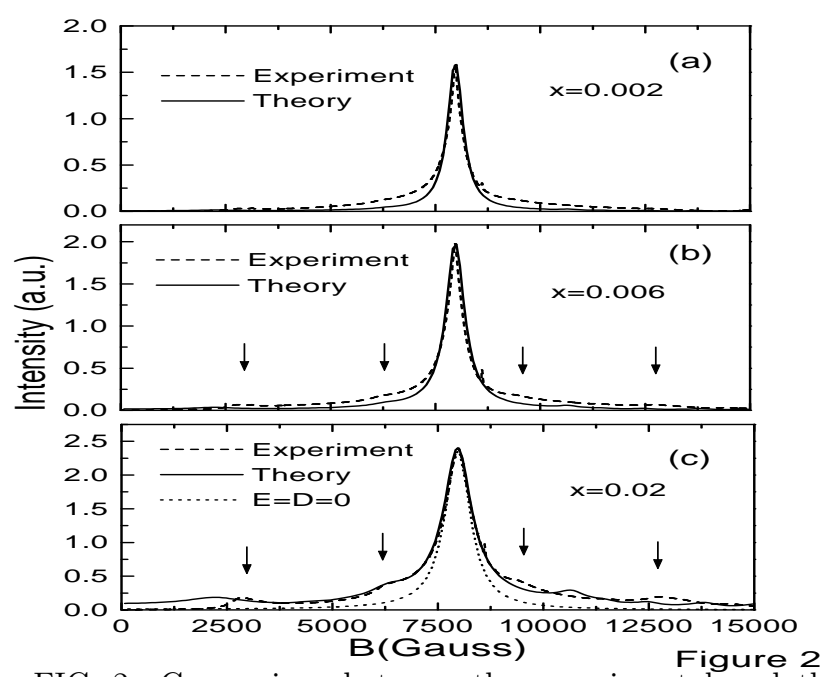

FIG. 2. Comparison between the experimental and theoretical EPR spectra for three different concentrations of $\mathrm{Mg}$, in the absence of interchain coupling. The values of $g^{a a}=2.254, g^{b b}=2.177$, and $g^{c c}=2.165$ are taken from Ref. [13]. The parameters of the Hamiltonian (Eq. 1) are taken from neutron scattering experiments 10,11: $J=280 K, D=-0.039 J, E=-0.127 J$. The arrows indicate the positions of the secondary peaks.

In Fig. 2 we show the experimental data 13 for $I(x, T)$ with three different values of $x \quad(x=$ $0.002,0.006$, and 0.02 ) at $T=2 \mathrm{~K}$ and compare them with the calculations. The uniform magnetic field $\mathbf{B}$ points in the $\mathbf{x}$ (or $\mathbf{c}$ ) direction, while the direction of the microwave field is $\mathbf{n}=\mathbf{z}$ (or $\mathbf{a}$ ). The width and intensity of the lorentzians corresponding to each transition have been adjusted considering the main peak. These are the only adjustable parameters. The integrated intensity is determined by $x$. The most prominent feature of Fig. 2 is the main peak at around $8000 \mathrm{G}$ which is related to very long chains for which the splitting induced by the anisotropy terms is much smaller than $g^{c c} \mu_{B} B$.
Surrounding the main peak there are some secondary peaks which correspond to the chains for which the magnitude of the anisotropy splitting is of the same order as $g^{c c} \mu_{B} B$. In order to clarify the last statement, let us consider the simplest case of $E=0$. The splitting $\Delta(N)$ (between the $S^{z}=0$ and the $S^{z}= \pm 1$ states) induced by the anisotropy term $D S_{z}^{2}(D<0)$ for odd and even chains is represented schematically in Fig. 3. With respect to these latter states, a uniform magnetic field $\mathbf{B}$ along the $\mathbf{x}$ direction (perpendicular to the chains), does not shift the $S_{x}=0$ state $((|11\rangle-|1-1\rangle) / \sqrt{2})$ but mixes $|10\rangle$ and $\frac{1}{\sqrt{2}}(|11\rangle+|1-1\rangle$ giving rise to a splitting between the lowest triplet states $\mathrm{T}_{1}$ and $S_{x}=0$ : $\delta(N)=\Delta(N) / 2+\sqrt{(\Delta(N) / 2)^{2}+\left(g^{c c} \mu_{B} B\right)^{2}}$. The application of a microwave magnetic field along the direction of the chains will induce transitions between these two states giving rise to resonant peaks when $\hbar \omega=\delta(N)$, being $\omega$ the microwave frequency. Then, chains larger than 40 sites $\left(\Delta(N)<<g^{c c} \mu_{B} B\right)$ contribute to the main peak centered at $\hbar \omega=g^{c c} \mu_{B} B$. For chain lengths between 15 and $30, \Delta(N)$ is of the same order as $g^{c c} \mu_{B} B$ giving rise to some secondary peaks at higher and lower energies than $g^{c c} \mu_{B} B . \Delta(N)$ is negative for odd chains and positive for even ones, so the peaks on the left side with respect to the main peak come from even chains while the ones on the right side are produced by odd chains. In Fig. 2 c) we have also plotted the absorption curve corresponding to no anisotropy $(D=E=0)$. As is expected from the analysis given above, no feature is observed apart from the main peak. We also note that the weight of the secondary peaks is bigger for larger doping concentration $(x=0.02)$. This is due to the fact

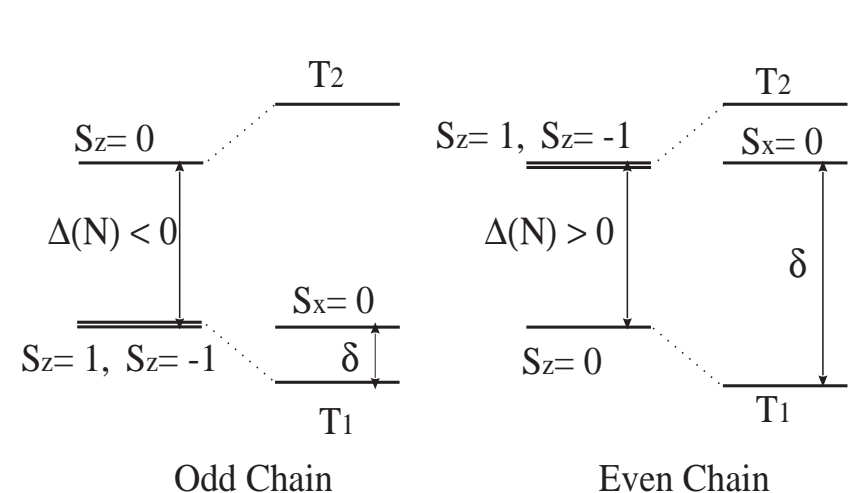

FIG. 3. Diagram which shows the effect on the triplet states of an applied magnetic field $\mathbf{B}$ in the $x$ direction in the presence of the anisotropy term $S_{z}^{2}$. 
The calculated spectra are very sensitive to the values of $D$ and $E$. However the best agreement is obtained using the experimental values given above [10,11. In order to show the sensitiveness of the spectra to these parameters, in Fig. 4 we plot the spectrum for $x=0.02$ and two different sets of parameters.

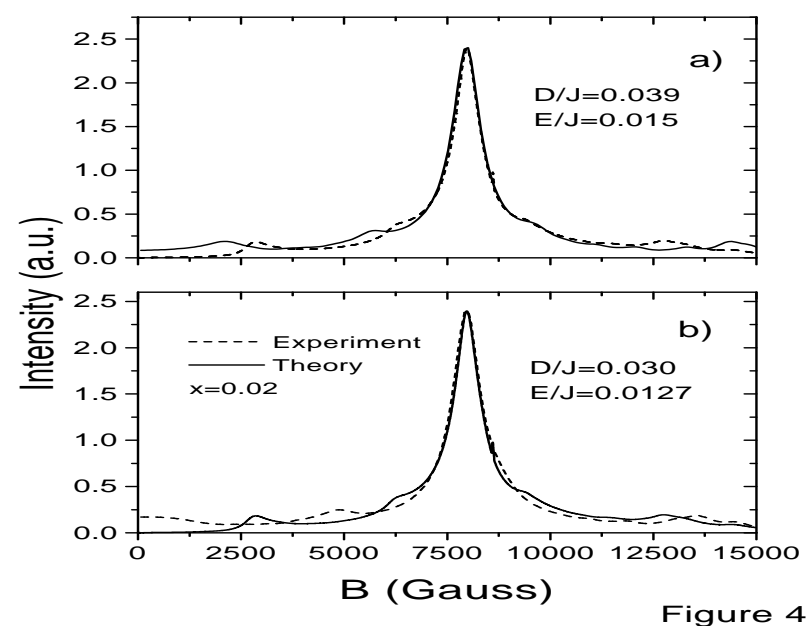

FIG. 4. same as Fig.2 for $x=0.02$ and a) $D=0.039 J$, $E=0.015 J$; b) $D=0.030 J, E=0.0127 J$.

The position of the different peaks is well reproduced within an error of $1000 \mathrm{G} \sim 1 K$ (the spike at around $8500 \mathrm{G}$ is a calibration feature). This error is of the same order of magnitude as the interactions neglected in our theory 16. It is important to note that there are no fitting parameters changing the positions of the secondary peaks. This agreement shows clearly that the secondary peaks of the EPR spectra are originated by the effect of spatial anisotropy on chains with lengths between 15 and 40 sites. Due to experimental difficulties at very low fields, there is an artificial cutoff below 2500G.

In Ref. [6], we have included the interactions between chain segments in order to reproduce the specific heat measurements of Ref. [9] at zero magnetic field. Because of this it is important to determine how sensitive the calculated EPR spectra are to the inclusion of these interactions. The origin and the magnitude of this $J^{\prime}$ inter-chain interaction is explained in Refs. [6, 15. Since a calculation of all possible $J^{\prime}$ s and their effect on a 2D (or 3D) topology is a formidable task, we model the effect of $J^{\prime}$ considering a collection of chain segments with effective 1D topology with exchange interaction $J^{\prime}$, coming from a uniform distribution between $-J_{\max }^{\prime}$ and $J_{\max }^{\prime}$. We obtain that the inclusion of realistic values of $J^{\prime}$ has no effect on the position of the peaks. The only effect of $J^{\prime}$, as might be expected, is a broadening of the secondary peaks and the corresponding small reduction of their maxima. As a consequence, the agreement between theory and experiment slightly improves for the peaks near $6000 \mathrm{G}$ and $12500 \mathrm{G}$.
In conclusion, by solving the low-energy spectrum of a Heisenberg Hamiltonian $H$ which includes experimental axial and planar anisotropy, we have reproduced in detail the low-temperature EPR data measured in $\mathrm{Y}_{2} \mathrm{BaNi}_{1-x} \mathrm{Mn}_{x} \mathrm{O}_{5}$ for different concentrations of $\mathrm{Mg}$. It is important to remark that there are no fitting parameters to reproduce the positions of the different peaks in the EPR spectra. These results confirm that the $S=1 / 2$ end chain excitations, which are asymptotically free for large chain segments, are experimentally observed. The fact that we have been able to fit all peaks with this model indicates that the $S=1 / 2$ feature observed by EPR experiments is unambiguously an end-chain excitation, ruling out any other possibility. Summarizing, the EPR experiments can be interpreted in terms of end states of an anisotropic Heisenberg model, and the discrepancies about the existence of spin $1 / 2$ excitations without classical analog are removed.

We thank C. Saylor for the experimental data. K.H. and C.D.B. are supported by CONICET, Argentina. A.A.A. is partially supported by CONICET. K. H. thanks the Max-Planck Institute PKS (Dresden) for computational facilities. This work was supported by PICT 03-00121-02153 of ANPCyT and PIP 4952/96 of CONICET.

[1] F.D.M. Haldane, Phys. Lett. 93A, 464 (1983); Phys. Rev. Lett. 50, 1153 (1983); J. Appl. Phys. 57, 3359 (1985).

[2] I. Affleck, T. Kennedy, E. Lieb, and H. Tasaki, Phys. Rev. Lett. 59, 799 (1987).

[3] T. Kennedy, J.Phys.Condens. Matter 2, 5737 (1990).

[4] S. Miyashita and S. Yamamoto, Phys. Rev. B 48, 913 (1993).

[5] S. White, Phys. Rev. Lett. 69, 2863 (1992); S. White and D.A. Huse, Phys. Rev B 48, 3844 (1993).

[6] C.D. Batista, K. Hallberg, A.A. Aligia , Phys. Rev B 58, 9248 (1998).

[7] S.H. Glarum, S. Geschwind, K.M. Lee, M.L. Kaplan, and J. Michel, Phys. Rev. Lett. 67, 1614 (1991).

[8] M. Hagiwara, K. Katsumata, I. Affleck, B.I. Halperin and J. Renard, Phys. Rev. Lett. 65, 3181 (1990).

[9] A.P. Ramirez, S-W. Cheong and M.L. Kaplan, Phys. Rev. Lett. 72, 3108 (1994).

[10] T. Sakaguchi, K. Kakurai, T. Yokoo, and J. Akimitsu, J. Phys. Soc. 65, 3025 (1996).

[11] G. Xu, J.F. Di Tusa, T. Ito, K. Oka, H Takagi, C.Broholm, and G. Aeppli, Phys. Rev B 54, R6827 (1996)

[12] O. Golinelli, Th. Jolicoeur and R. Lacaze, Phys. Rev B 45, 9798 (1992).

[13] C. Saylor, S. Geschwind, L. Desmarias, and S.-W. Cheong, preprint. 
[14] E.H. Lieb and D.C. Mattis, J. Math. Phys. 3, 749 (1962).

[15] C.D. Batista, A.A. Aligia and J. Eroles, Europhys. Lett. 43, 71(1998).

[16] The exchange interaction pependicular to the chains gives rise to random ferro or antiferromagnetic interactions between some $\mathrm{S}=1 / 2$ end states $[6]$. 